\title{
Frontières
}

\section{Apocalypses et fins de monde : enjeux de santé mentale}

\section{Viviane Annie Lew}

Volume 25, numéro 2, 2013

Apocalypses et imaginaires de la fin

URI : https://id.erudit.org/iderudit/1024939ar

DOI : https://doi.org/10.7202/1024939ar

Aller au sommaire du numéro

Éditeur(s)

Université du Québec à Montréal

ISSN

1916-0976 (numérique)

Découvrir la revue

Citer cet article

Lew, V. A. (2013). Apocalypses et fins de monde : enjeux de santé mentale. Frontières, 25(2), 56-74. https://doi.org/10.7202/1024939ar

\section{Résumé de l'article}

La problématique apocalyptique n'est pas sans interpeller le champ de la santé mentale. Les idéologies dans de nombreuses sectes s'appuient sur des images et des interprétations bibliques mais aussi des représentations post-modernes faisant référence aux extraterrestres et aux OVNI. Leurs leaders charismatiques présentent aussi des profils psychologiques problématiques qui peuvent les entraîner, tout comme leurs adeptes, à des conduites extrêmes. Les inquiétudes liées aux innovations technologiques comme les armes nucléaires, la détérioration de l'environnement de même que les annonces médiatiques peuvent entrainer des réactions anxiogènes qui affectent le bien-être des personnes. Les délires incluant des références apocalyptiques et de fin de monde se retrouvent aussi chez des patients souffrant de troubles psychotiques. Ces enjeux sont discutés en s'appuyant sur des exemples historiques et contemporains. 


\section{APOCALYPSES ET FINS \\ DE MONDE : ENJEUX DE SANTÉ MENTALE}

Viviane Annie Lew, M.D. Psychiatre

La croyance forte ne prouve que sa force, et non la vérité de ce qu'on croit

Frédéric Nietzche

RÉSUMÉ

La problématique apocalyptique n'est pas sans interpeller le champ de la santé mentale. Les idéologies dans de nombreuses sectes s'appuient sur des images et des interprétations bibliques mais aussi des représentations post-modernes faisant référence aux extraterrestres et aux OVNI. Leurs leaders charismatiques présentent aussi des profils psychologiques problématiques qui peuvent les entraîner, tout comme leurs adeptes, à des conduites extrêmes. Les inquiétudes liées aux innovations technologiques comme les armes nucléaires, la détérioration de l'environnement de même que les annonces médiatiques peuvent entrainer des réactions anxiogènes qui affectent le bien-être des personnes. Les délires incluant des références apocalyptiques et de fin de monde se retrouvent aussi chez des patients souffrant de troubles psychotiques. Ces enjeux sont discutés en s'appuyant sur des exemples historiques et contemporains.

MOTS-CLÉS: Apocalypse - santé mentale - délires - sectes - violence - suicide - anxiété

\section{ABSTRACT}

Apocalyptic representations are relevant in the field of mental health. The ideologies in numerous sects are based on images and interpretations fed by biblical references, but also by postmodern representations such as extraterrestrials and UFOs. These charismatic leaders present problematical psychological profiles which can carry them, as their followers, to extreme behaviors. Anxieties associated to technological innovations such as nuclear weapons, deterioration of the environment, as well as the 
media announcements can provoke stressful reactions, affecting the wellbeing of individuals. Delusions including apocalyptic and end of the world references are often reported among patients with psychotic disorders. These issues are discussed using historic and contemporary examples.

KEYWORDS: Apocalypse - mental health - delusions - sects - violence - suicide - anxiety

Les idéologies apocalyptiques et de fin de monde occupent une place importante dans l'histoire humaine et en particulier dans le monde occidental avec le fameux texte de l'Apocalypse de Jean de Patmos qui définit le scénario et les symboles les plus connus du déroulement de la fin des temps. Ces images et ces références serviront de modèle et de guide dans les développements ultérieurs et seront repris par les nombreuses sectes qui se réclameront de ce mythe auquel viendront s'articuler d'autres références plus actuelles, politiques, scientifiques et pseudoscientifiques. Ce maintien à travers le temps suggère que ces idées ont une fonction existentielle profonde. Elles interpellent à plus d'un titre les intervenants en santé dans la mesure où elles peuvent entraîner des problèmes de santé mentale à différents niveaux. En premier lieu, les enjeux de santé mentale sont associés aux groupes sectaires où domine une idéologie apocalyptique. Dans ces groupes, les leaders charismatiques aux traits de personnalité problématiques peuvent amener leurs fidèles, manipulés psychologiquement, à poser des gestes extrêmes, suicide ou attentats. En second lieu, les innovations technologiques comme la bombe nucléaire, les prédictions scientifiques entourant la détérioration de l'environnement et du climat, ou les prophéties diffusées par des sources pseudoscientifiques, répercutées par les médias sensationnalistes, peuvent jouer sur les peurs et les anxiétés diffuses dans les populations, affecter leur bien-être et, dans certains cas, demander une intervention thérapeutique. En dernier lieu, les délires apocalyptiques se retrouvent chez des patients traités pour des troubles mentaux, ce qui demande de cerner leur signification dans leur économie psychique. Nous illustrerons successivement chacun de ces points à partir d'une recension des travaux effectués dans ces domaines.

\section{LES GROUPES SECTAIRES ET LES DISCOURS APOCALYPTIQUES ET DE FIN DE MONDE}

Les représentations et les interprétations apocalyptiques des évènements naturels et sociopolitiques annonçant la fin du monde et l'intervention divine constituent des idées forces présentes dans I'histoire de I'Occident, suite à l'influence des textes bibliques et évangéliques, entre autres, le texte de l'Apocalypse de Jean de Patmos qui, dans ses visions, décrit les cataclysmes qui s'abattront à la fin des temps avant le retour du Christ et l'avènement du 
Royaume de Dieu. Ce scénario fondamental alimente les interprétations subséquentes qui tenteront de déchiffrer les symboles et de fixer la date précise de la fin du monde à partir de combinaisons numérologiques dont on trouve des essais dans I'histoire des idées apocalyptiques qui ont subsisté malgré les interdits des institutions ecclésiastiques. Les approches rationalistes, qui se mettent en place à l'ère des Lumières, taxeront ces croyances d'obscurantistes (Weber, 1999) et la sécularisation n'empêchera pas le maintien des idées apocalyptiques qui tendent à se diversifier dans le monde contemporain. À côté des sectes évangéliques nourries des textes et des interprétations bibliques, les groupes sectaires post-modernes se multiplient, fondant leur contenu doctrinal sur des perspectives New Age liés aux OVNI et aux extra-terrestres. Ces thématiques ne se situent pas toujours dans des catégories mutuellement exclusives mais elles peuvent se conjuguer dans des assemblages hétéroclites, des bricolages qui se déclinent de façon variée. Elles véhiculent des idées irrationnelles alimentant les convictions profondes ancrées chez les leaders et les adeptes de ces cultes, en attente de leur réalisation imminente, mais toujours retardée. Le maintien des idéologies apocalyptiques dans le temps suggère qu'elles remplissent une fonction existentielle importante, reflétant les angoisses et les peurs réactivées par les catastrophes naturelles et les crises sociopolitiques dont l'histoire occidentale est remplie. Weber souligne les fonctions apaisantes des prédictions apocalyptiques: «Qu'elles aient été prononcées avant le Christ ou depuis, les prophéties apocalyptiques sont des tentatives pour interpréter leur époque, pour réconforter et guider, pour décrypter le sens du présent et de l'avenir. Elles rattachent typiquement la crainte à l'espoir: adversités et horreurs vont instaurer la félicité publique et privée, l'absence de toute souffrance et de tout mal. » (1999, p. 39-40) Mais dans les cas extrêmes, elles peuvent au contraire être source de manipulation, de contraintes psychologiques, de culpabilité et mener à des conduites désespérées et mortifères, comme le montrent de nombreuses occurrences historiques et contemporaines dont nous présenterons quelques exemples.

Au plan historique, l'exemple des Vieux-Croyants russes apparus au XVII e siècle (Robbins, 1986) met en relief les conséquences des attentes apocalyptiques qui se basent sur les croyances véhiculées dans le Livre de Cyrill, un recueil de textes apocalyptiques du sud de I'Ukraine. On y retrouve la figure de l'Antéchrist, mais la seconde venue du Christ n'est pas soulevée. Plusieurs groupes de croyants appartenant à des ordres monastiques, se considérant comme les seuls gardiens de la tradition, s'opposèrent aux innovations ecclésiastiques visant à des modifications des rituels liturgiques pour les adapter à ceux des Églises orthodoxes grecque et ukrainienne. Ils se séparèrent alors de l'Église officielle, un schisme auquel de nombreux paysans, inquiets des tendances bureaucratiques tsaristes et attachés aux rites jusque-là pratiqués, se rallièrent. Pour les Vieux-Croyants, le Tsar de Russie et le chef de l'Église orthodoxe russe, desquels émanaient ces changements, étaient associés à l'Antéchrist. La preuve de l'inscription de cet événement 
dans le plan divin et satanique était confirmée par la date du concile décisionnaire qui s'était réuni en 1666. Cette date reprenait le nombre 666 associé à celui de la Bête, décrit dans I'Apocalypse. Comme Robbins (1986) le souligne, cette idéologie où s'entrelacent des motifs politiques et religieux menèrent à long terme à des suicides de masse :

Imprisoned in the Third Rome mystique and the orthodox assumptions about the theocratic role of the tsar, the Old Believers were pushed to elevate their opposition to [...] reforms to the level of a subversive political theology, and ultimately to entertain extreme apocalyptic notions which implied the hopelessness of the worldly situation dominated by Antichrist. Moods of both apocalyptic despair and political truculence were intensified by a sequence of ups and downs [...]. A feedback process of "deviance amplification" [...] or interaction between escalating persecution and intensifying alienation and deviant protest is clearly evident and culminated in a great wave of mass suicides. (1986, p. 8)

Les historiens rapportent ainsi le recours au suicide par auto-immolation, entre la fin du XVII e siècle et le XIXe siècle, de dizaines de milliers d'adeptes. Si au départ les attentes apocalyptiques affirmant que l'Antéchrist avait déjà pris le pouvoir et que la fin du monde était imminente demandaient l'adoption de conduites ascétiques exemplaires, pour certains croyants elles étaient considérées comme insuffisantes. Prenant modèle sur les premiers martyrs chrétiens, ils préconisaient l'immolation par le feu comme mode de purification. Cette pratique, qui ne faisait cependant pas l'unanimité, devint plus répandue avec l'amplification des idées apocalyptiques suite à la répression politique et militaire qui confirmait leur conviction d'être persécutés par le pouvoir central tant civil que religieux. Passés à la clandestinité, des membres de ce mouvement se réfugièrent dans des zones isolées de la Russie pour rompre avec un monde considéré comme contaminé et condamné. Lorsque leurs colonies et leurs monastères se voyaient entourés par les forces armées et sur le point d'être investis, les suicides de masse survenaient alors. Cependant, pour des observateurs, ces incidents étaient volontairement provoqués par les Vieux-Croyants pour accéder au martyre et hâter la fin du monde.

Les groupes sectaires contemporains se fondent aussi sur un dispositif doctrinal mêlant les arguments politiques et religieux et recourent souvent au suicide collectif comme modalité de réalisation des prédictions apocalyptiques. Un premier exemple est fourni par la Branch Davidians, dont l'idéologie se base sur la reprise du scénario proposé dans l'Apocalypse de Jean de Patmos. Dans cette secte dissidente de l'Église Adventiste du $7^{\text {e }}$ jour, dirigée par David Koresh à partir de 1987, le leader est considéré comme le Christ annoncé dans le texte apocalyptique et ses paroles ont force de révélation. David Koresh, reprenant les concepts fondamentaux des textes bibliques, confirme que ses adeptes ont un statut de peuple choisi par Dieu qui en fait le fondement de son Église (Office of Special Counsel, s.d). Il proclame qu'il a toute autorité pour révéler les secrets des Sept Sceaux inclus dans le texte de 
l'Apocalypse qui établit une séquence finale, allant de la fin des temps à la vie éternelle en passant par le Jugement Dernier. Décrivant les tribulations qui I'attendent, ainsi que son groupe, il annonce qu'ils seront impliqués dans une guerre qui surviendra au Moyen-Orient ou en Israël et qui sera suivie de la fin du monde. Confrontés à leurs ennemis, Babylone ou la Bête - des images majeures dans le texte - mais aussi au gouvernement américain, il prophétise que les Davidians feront l'objet d'attaques et qu'ils seront décimés par les «Babyloniens», en plusieurs phases. Ils devront accepter de lutter et de mourir pour leur Dieu et leur leader et se préparer à défendre leur domaine par les armes, face à une puissante armée. Le Sixième Sceau sera ouvert lors du retour glorieux de David Koresh, qui sauvera les saints et détruira les méchants, annonçant le Jugement Dernier. Quant au Septième Sceau, il annonce que la fin du monde surviendra dans un cadre conjuguant des éléments naturels et humains, la grêle et le feu mêlé au sang. Le thème du feu, un moyen requis pour accéder au Paradis lors de la bataille contre les forces de Babylone, est central dans ces enseignements qui reprennent des citations de prophètes à ce sujet. Ces commentaires du texte apocalyptique visent à confirmer le statut spécial du groupe en reprenant la rhétorique de l'Apocalypse et ses images principales et en les calquant sur les préoccupations contemporaines liées la situation problématique de cette secte dans l'espace américain.

L'importance symbolique du feu se concrétisera par la mort dans un incendie, intentionnel ou accidentel, du leader et de nombreux adeptes ( 76 personnes dont 20 enfants et des femmes enceintes) suite à l'attaque de la communauté installée dans une propriété près de Waco, au Texas, par des forces gouvernementales, en 1993. Les autorités avancèrent plusieurs motifs pour expliquer leur assaut: préoccupations quant à la santé mentale du leader et des adeptes, accusations d'abus sexuels de mineurs, trafic d'armes et menaces à I'ordre public; des raisons qui firent l'objet par la suite de multiples polémiques quant à leur bien-fondé (Wright, 1995; Newport, 2006). Le siège dura 51 jours avant l'assaut final et l'incendie. Ce dénouement fut interprété comme un suicide de masse (Dein et Littlewood, 2000), une auto-immolation (Adityanjee, 1994) qui s'inscrirait dans «une réactualisation du scénario de l'Apocalypse johannique» (Prévost, 1999, p. 159) pour confirmer la prophétie apocalyptique de Koresh à l'effet que son groupe serait attaqué par le gouvernement américain, la Babylone contemporaine.

Dans le cas du groupe sectaire du Temple du Peuple et de son leader, Jim Jones, les textes et les discours révèlent des strates idéologiques multiples, à la fois bibliques et contemporaines (Schuyler et Trinh, 2000). Aux perspectives apocalyptiques émanant des mouvements pentecôtistes s'ajoutent des références aux idées sociales libérales et méthodistes ainsi qu'à des perspectives communistes. Les sermons du leader prédisant une catastrophe génocidaire s'accompagnent de la narration de visions religieuses extrêmes où dominent les images de la fin du monde associée à l'annihilation nucléaire, aux tremblements de terre et aux révolutions politiques fascistes liées aux 
mouvements nazis et au Klux Klux Klan. Selon Robbins, ces idées donnent lieu à des tensions entre deux perspectives opposées:

Jones' prophecy converged with that of the tribulationists in terms of a vision of an imminent holocaust amidst a triumph of evil and corruption in the world. [...] Jones' prophecy was far more radical than those of contemporary Adventist groups: he focused on imminent apocalyptic disaster rather than on Christ's millennial salvation, and his eschatology, therefore, had to resolve a choice between apocalyptic struggle with "the beast" or collective flight to establish a post-apocalyptic kingdom of the elect. Until the end, the People's Temple was directed toward the latter possibility. (1986, p. 13)

Pour échapper aux critiques et aux attaques médiatiques, politiques et juridiques des opposants américains à la secte et pour éviter les radiations nucléaires d'une guerre mondiale annoncée, les adeptes s'installèrent en 1977 à Jonestown, en Guyane Britannique, où ils créèrent un sanctuaire agricole, sans pouvoir cependant échapper à la surveillance des autorités américaines. En 1978, des représentants du Congrès américain, des journalistes et des parents préoccupés par la situation de leurs enfants dans la secte vinrent enquêter sur la commune, mais cette visite provoqua une fusillade qui entraîna la mort de députés. Jim Jones informa alors la communauté qu'il ne voyait pas comment elle pourrait mener une vie normale face aux exigences d'un gouvernement américain démoniaque (Dein et Littlewood, 2000). Il préconisa un suicide collectif par ingestion de poison et de tranquillisants, comme acte révolutionnaire - une solution à laquelle le groupe semble avoir adhéré - , entraînant la mort de près de 1000 personnes, dont 260 enfants. Ce suicide a été interprété par Black (1990) comme obéissant à des raisons à la fois altruistes (le bien du groupe étant jugé supérieur au bien-être personnel) et fatalistes (à cause du désespoir associé à l'incapacité de lutter contre un environnement hostile), ce dernier motif étant privilégié par Dein et Littlewood (2000). La mort de Jim Jones, quant à elle, obéirait à d'autres motifs, comme le souligne Baechler:

Dans son suicide, il est clair qu'il faut retenir une dimension de fuite hors d'une situation jugée insupportable; il n'est pas exclu qu'il ait cherché à se punir de son échec. En ne mourant pas seul, il n'a pas seulement voulu mourir tout entier. Il est possible qu'il ait aussi succombé au vertige d'Érostrate et ait voulu perpétuer sa mémoire par un crime énorme, faute de réussir un exploit mémorable. (Baechler, cité dans I’Encyclopédie sur la mort, 2012)

Une autre secte, I'Ordre du Temple Solaire, révèle une autre configuration idéologique où les idées apocalyptiques chrétiennes, plutôt secondaires, sont associées à des références à la tradition rosicrucienne, un ordre mystique orienté vers le cheminement spirituel. À cette influence s'ajoutent des interprétations numérologiques et des références aux idées du New Age inspirées des prises de position écologistes annonçant des cataclysmes liés à la détérioration rapide de la terre et sa destruction, à laquelle survivraient les adeptes. 
Dans les années 1990, cette secte connût une remise en question du charisme des leaders et de nombreuses tensions internes amplifiées par des enquêtes policières internationales qui ont contribué à nourrir des tendances paranoïaques. Entre 1994 et 1997, des meurtres, des suicides et des incendies surviennent dans plusieurs pays (Québec, Suisse, France), des incidents qui culminent dans la mort de 74 personnes dont celle de deux leaders. S'agit-il de meurtres ou de suicides volontaires ou imposés? Il est difficile de répondre à la question, mais il semblerait que pour ces adeptes la mort contribuerait au transit vers un autre univers ou vers la planète Sirius et l'atteinte d'un statut surhumain, I'existence sur terre devenant de plus en plus inacceptable (Infosecte, 2003; Lewis, 2006; Encyclopédie de la mort, 2012).

Les idées de migrations vers d'autres univers se retrouvent dans l'idéologie apocalyptique post-moderne de la secte Heaven's Gate, où les doctrines rompent avec les perspectives bibliques traditionnelles pour proposer un «matérialisme technologique» avancé reflétant une herméneutique biblique extraterrestre (Zeller, 2010). Les discours insistent sur la détérioration de l'état de la planète et son recyclage imminent et s'arriment à des croyances issues du New Age associées aux mythes de la visite des OVNI et d'extraterrestres que les humains spirituellement évolués iront rejoindre:

Aliens planted the seeds of current humanity millions of years ago, and have to come to reap the harvest of their work in the form of spiritual evolved individuals who will join the ranks of flying saucer crews. Only a select few members of humanity will be chosen to advance to this transhuman state. The rest will be left to wallow in the spiritually poisoned atmosphere of a corrupt world. (Lewis, 2003, p. 133)

Cette perspective peut expliquer le suicide, en 1997, d'un certain nombre d'adhérents convaincus qu'ils seraient transportés dans un vaisseau spatial situé près de la comète Hall-Bopp, qui passait non loin de la terre à ce moment, pour rejoindre un univers surhumain (Davis, 2000).

Les conceptions apocalyptiques ne se limitent pas au monde occidental, comme le montre l'exemple de la secte japonaise Aum Shirinkoyo (religion de la suprême vérité), qui prévoyait la fin du monde pour le début du XXI siècle. Son idéologie amalgame des influences provenant des textes apocalyptiques avec des références à la guerre finale entre les forces du Bien et du Mal (ou à la bataille d'Armageddon), auxquelles s'ajoutent celles du bouddhisme tibétain (réincarnation), de l'hindouisme (avec une fixation en particulier sur le dieu de la destruction, Shiva), du shintoïsme, des prophéties de Nostradamus, des romans de science-fiction d'Asimov et des croyances New Age (Senate Government Affairs Permanent Subcommittee on Investigations, 1995; Hudson, 1999). Ce syncrétisme est complété par des analyses économicopolitiques prédisant l'effondrement du Japon et sa disparition, ainsi qu'une guerre finale à laquelle ne survivront que les adeptes. La secte n'hésite pas à recourir à des méthodes violentes terroristes (Danzig et al., 2011), allant jusqu' à mettre en place une escouade d'assassins chargés de kidnapper et de tuer les opposants et les critiques. Dès 1990, la secte planifie d'acquérir 
des armes de destruction de type biologique (botulisme, anthrax, sarin, tabun) utilisées à diverses occasions avant la grande attaque du 20 mars 1995 dans le métro de Tokyo, où le sarin provoqua la mort de onze personnes et affecta la santé de 5000 personnes. Cette stratégie terroriste était préconisée comme guérilla défensive nécessaire pour se protéger de l'Armageddon, mais aussi pour dissuader les autorités de continuer à enquêter sur la secte et arrêter ses opposants. Arrêté avec un groupe de ses fidèles, son leader fut accusé de meurtre et condamné à mort.

Ces perspectives apocalyptiques et de fin de monde empruntent donc leur contenu à des sources diverses et annoncent une catastrophe proche en la fondant sur des arguments tant religieux que modernes, dans des configurations hétérogènes, incohérentes et irrationnelles. Mais leur irrationalité n'empêche pas qu'elles soient l'objet de convictions profondes sincères et partagées par des adeptes. Ceux-ci peuvent aussi éprouver des doutes non exprimés pour ne pas risquer de subir les sanctions des instances de contrôle de la secte ou être victimes de délation de la part des membres de l'entourage. Cette stratégie conforte ainsi une image de conformité dans des contextes où la critique ou la remise en question des croyances sont des manifestations réprimées. Le rapide survol des formes de violence et de suicide dans ces groupes met en évidence les extrêmes auxquels les idéologies qui leur sont rattachées peuvent amener leurs leaders et leurs membres lorsque, comme le soulignent Dein et Littlewood (2000), elles se conjuguent à une philosophie dualiste opposant le bien et le mal, à une emprise totale du leader et à une isolation du groupe.

\section{LES GROUPES SECTAIRES ET LES ENJEUX DE SANTÉ MENTALE}

Comme I'ont montré nos exemples, les leaders de ces groupes sectaires ont une emprise redoutable sur leurs fidèles et plusieurs travaux ont été consacrés à dégager leur profil psychologique. Les biographies mettent en évidence, selon Dein et Littlewood, des traits communs: «an absent father from a young age, solitude in childhood, intolerance of criticism, a personal revelation and the presence of narcissistic and paranoid personality traits 》 (2000, p. 110). On note aussi des éléments de grandeur et de mégalomanie associés à une personnalité charismatique et autoritaire. Ces personnes s'identifient à des figures religieuses ou surnaturelles majeures qui les habitent ou les inspirent, ou dont ils sont la réincarnation. Ils en tirent la source de leur autorité et le signe de leur statut supérieur, reconnu par leurs adeptes.

La biographie de David Koresh, le leader de la Branch Davidian, fait état de problèmes rencontrés dans son développement psychosocial (mère adolescente, père absent, beau-père sévère, abus sexuels, décrochage scolaire). L'aspect grandiose de sa personnalité se manifeste par son identification à l'Agneau de Dieu, le Messie venu libérer l'humanité de ses péchés, qui ouvre les sept sceaux mentionnés par Jean de Patmos, révélant qu'il est le Christ 
que l'Apocalypse annonce, monté sur un cheval blanc et brandissant une épée. Affirmant que l'esprit de Jésus de Nazareth I'habite depuis 1985, il se considère comme le septième et dernier prophète, le sauveur du peuple de Dieu qui joue un rôle central dans le processus eschatologique cosmique et qui a toute autorité pour innover à partir du texte biblique, décevoir les gens et leur mentir, afin de sauver ses adeptes (Office of Special Counsel, s.d.).

On retrouve des traits de grandeur semblables dans le profil de Jim Jones, le dirigeant du Temple du Peuple. Dès le début de sa carrière, il se proclame la réincarnation d'Akhenaton, de Bouddha et de Lénine (Harvey, 2009) et prétend occuper la place de Jésus et être le seul Dieu visible. Avec le temps, une détérioration de sa personnalité se précise, associée semble-t-il à une consommation de divers médicaments dont il aurait été dépendant (Rosenberg, s.d.).

Les leaders de I'Ordre du Temple Solaire sont, quant à eux, convaincus d'avoir des pouvoirs médiumniques et thérapeutiques (Lewis, 2006) et d'avoir été choisis par des entités divines qui leur communiquent des messages (Infosecte, 2003). Les dirigeants du groupe Heaven's Gate, comme Marshall Applewhite et Bonnie Nettles, se considèrent comme des surhumains provenant du paradis et qui se sont incarnés dans des corps humains pour apporter leur message aux terriens (Grunschloss, 1998; Davis, 2000). Quant à Shoko Asahara, le dirigeant de la secte Aum Shirinkoyo, il professe avoir des habiletés surnaturelles et affirme sa grandeur en se déclarant la réincarnation du dieu Shiva puis de Jésus-Christ (Butz, 2000). Exhibant des tendances paranoïdes de plus en plus évidentes suite aux déboires politiques expérimentés par la secte, il les attribue à un vaste complot de la part de divers groupes (Juifs, gouvernements américain et japonais, francs-maçons), confirmé par sa défaite aux élections japonaises. Celle-ci constituait la preuve que les forces du Mal opposées à son projet étaient à l'œuvre et cette situation exigeait le recours à des actions terroristes pour pouvoir les vaincre.

Ces leaders font preuve d'un charisme évident et sont ainsi capables de convaincre leurs adeptes, qui dans certains groupes se comptent par milliers, de leur statut extraordinaire et de leurs pouvoirs. Cette emprise sur le groupe, qui renvoie à une «éthique de l'obéissance radicale» (Davis, 2000, p. 249), est maintenue grâce à des techniques de manipulation diverses (Dein et Littlewood, 2000; Schuyler et Trinh, 2000; Whitsett et Kent, 2003) : recours à la domination tant homosexuelle qu'hétérosexuelle des fidèles par les leaders ou, au contraire, à des formes d'ascétisme sexuel pouvant aller jusqu'à des mutilations. Dans le cas de la secte Heaven's Gate, la castration a été adoptée par un leader et plusieurs hauts dirigeants pour éliminer le désir sexuel, considéré comme une pulsion malsaine qui disparaîtra lorsque la surhumanité sera atteinte (Muesse, 1994).

La séparation des adeptes de la secte du reste de la société, en préconisant la formation de communes, la rupture de toutes les relations familiales et amicales et le contrôle des fréquentations servent aussi à réduire les influences extérieures et les possibilités de fuite, tout en augmentant la dépendance au 
groupe qui devient à la fois source de soutien et de soupçon. L'insistance sur l'opposition entre «eux» et «nous» par la diabolisation des étrangers et des instances gouvernementales vient renforcer les tendances paranoïdes. Ce système de contrôle fait appel à des techniques variées (influence, persuasion, délation, surveillance des familles, des idées et des comportements, direction de conscience, punitions) et concourt à provoquer des sentiments d'humiliation et de culpabilisation qui affectent le sens critique.

Ces dispositifs contribuent-ils de façon significative à une détérioration de la santé mentale des adhérents confrontés à des conditions de stress intérieurs et extérieurs importants? À cet égard, les recherches, peu nombreuses, en arrivent à des conclusions mitigées, faute de données fiables. Pour des chercheurs, les contextes sectaires joueraient de façon négative sur le bien-être psychologique des adultes, mais surtout celui des enfants dont le développement psychosexuel serait affecté (Whitsett et Kent, 2003). D'autres auteurs (Dein et Littlewood, 2000) sont d'avis que les taux de maladie mentale chez les membres de culte ne seraient pas plus élevés que dans la société en général, mais ils suggèrent que certains des adeptes «possess borderline traits and use psychological defences such as splitting, idealisation and projective identification» (p. 110). Une recension critique des recherches dans ce domaine (Aronoff, Lynn et Malinoski, 2000) en dégage les conclusions. Les individus qui adhèrent à une secte ne présenteraient pas nécessairement de symptômes psychopathologiques particuliers, mais ils peuvent cependant être cachés étant donné les contraintes environnementales et les pressions à la conformité exercées par l'environnement sectaire. Une fois sortis de cet environnement, les ex-membres peuvent vivre des difficultés d'adaptation à leur nouvelle vie. Néanmoins, ces études ne portent pas spécifiquement sur les sectes apocalyptiques et de fin de monde qui peuvent présenter des contraintes susceptibles d'influencer la santé mentale de façon plus marquée.

D'autres formes extrêmes de conduites liées en particulier aux suicides de masse et aux actions terroristes suggèrent cependant que les dimensions psychopathologiques sont plus notables. Moins étudiées sont les répercussions psychologiques sur les fidèles appartenant à ces sectes, lorsque les prédictions apocalyptiques ne se réalisent pas. Une recension des recherches sur cette thématique (Dawson, 1999) indique que les dissonances cognitives provoquées par cette situation donnent lieu à des modalités adaptatives diverses selon les groupes. Alors que certains groupes disparaissent, d'autres survivent et commencent ou continuent à faire du prosélytisme ou, au contraire, réduisent ou cessent cette stratégie de recrutement.

Cet ensemble de recherches met en évidence la dynamique interne aux groupes et la complexité des rapports entre les leaders et leurs fidèles qui renvoient à des modalités de contrôle et de séduction. Comme le souligne Torkghashghaei, ce mécanisme de séduction permet aux adeptes de pouvoir «échapper aux angoisses, souffrances, à la dépression et aux douleurs insupportables. La solution psychotique est de se transformer à la manière d'une résurrection ou renaissance dans un Autre qui a un monde plus 
séduisant» (2009, p. 222). Les modalités hypnotiques, de régression et de transfert contribuent à la soumission des adeptes et à leur abandon de la responsabilité au profit d'une soumission qui les amènerait à renoncer à leur individualité pour se fondre dans la secte, qui devient alors le porteur des normes à suivre.

\section{LES HORIZONS DE FIN DE MONDE ET LA SANTÉ MENTALE}

Les préoccupations apocalyptiques ou de fin de monde ne sont cependant pas I'apanage des sectes; elles peuvent aussi faire partie de I'horizon socioculturel à des moments historiques ou à la suite d'évènements spécifiques (catastrophes, épidémies) et se diffuser alors à l'ensemble social. C'est le cas dans le contexte contemporain où les innovations scientifiques et technologiques peuvent provoquer des peurs relayées par les films, les médias et les nouvelles technologies de communication, alimentant des représentations catastrophiques dans la culture populaire qui peuvent provoquer des réactions anxieuses et jouer sur le bien-être physique et mental de segments particulièrement sensibles de la population.

Nombreuses ont été les sources de préoccupations apocalyptiques de ce type dans le monde occidental. On retrouve en premier lieu, les peurs associées aux dates des millénaires. Si l'existence des peurs de l'an mille ont fait l'objet de polémiques entre historiens, certains tentant d'en démontrer la véracité (Landes, 1996), d'autres les remettant totalement en question (Gouguenheim, 1999), celles plus proches de nous, au tournant de I'an 2000, ont été mieux cernées. Les prédictions entourant l'effondrement des systèmes informatiques, avec le bogue de l'an 2000, et leurs répercussions sur les communications et l'économie mondiale avaient ainsi provoqué des inquiétudes (approvisionnement en nourriture, en eau et en sources d'énergie) tant parmi la population que les autorités (plans d'urgence). Par exemple, selon un sondage américain réalisé en 1999 (CNNUS, 1999), 53\% des répondants s'attendaient à un bris informatique majeur dans le système bancaire et près de $50 \%$ prévoyaient retirer plus d'argent de leur compte; $38 \%$ s'attendaient à des émeutes ou d'autres formes de troubles sociaux; $33 \%$ prévoyaient stocker des aliments, $23 \%$ du carburant, $13 \%$ comptaient s'armer et près de $10 \%$ s'attendaient à la fin du monde au tournant du siècle. En France, selon un autre sondage (IPSOS, 1999), 18\% rapportaient des peurs liées au passage à I'an 2000, associées en particulier à une guerre mondiale (31\%) et à une panne informatique (16\%).

À part les millénarismes, l'occurrence d'épidémies, comme la Peste noire (Delumeau, 1978) avait aussi été associée à l'apocalypse et provoqué de nombreuses peurs, réactivées par l'épidémie du VIH/sida dans ses premiers temps (Voir Lévy, dans ce numéro), les préoccupations diminuant avec les innovations médicales et pharmacologiques. Néanmoins, une proportion encore importante de personnes continue d'exprimer des craintes face à ce 
syndrome. Par exemple, selon un récent sondage mené en île de France, 27\% des répondants disaient craindre «beaucoup ou pas mal» le sida (L'Express, 2011). D'autres épidémies comme la grippe espagnole et, plus récemment, celle du H1NI, avaient aussi provoqué des états de panique dans de nombreux pays, associés aux projections épidémiologiques et aux déclarations de I'OMS qui avait lancé un processus de vaccination mondiale (Sieber, 2010).

Aux épidémies, il faut ajouter les craintes de l'apocalypse nucléaire, surtout aux États-Unis, suite à la course aux armements nucléaires. Dans les années 1950, cette menace donna lieu à des plans de défense civile comprenant la construction d'abris atomiques bien pourvus en provisions et des exercices de prévention répétés. Au début des années 1960, des sondages indiquent que $30 \%$ des Américains interrogés avaient peur des conséquences d'une guerre nucléaire et $11 \%$ avaient des difficultés à faire des plans pour l'avenir (Lyons, 2003). L'anxiété nucléaire était particulièrement présente chez les 13-17 ans qui étaient soumis à des exercices de préparation aux effets de la guerre nucléaire dans les écoles et ce, chaque mois. Si ces craintes ont significativement diminué par la suite, elles continuèrent néanmoins à subsister de façon épisodique, se réactivant à l'occasion d'évènements comme la panne électrique majeure de 1965 ou de guerres régionales (Boyer, 1984).

Plus récemment, un ensemble d'études sur la peur de la guerre nucléaire parmi les jeunes adultes finlandais indique qu'elle affecterait plusieurs aspects de la santé mentale: stress, dépression, incapacité à affronter les problèmes quotidiens, insomnie, anxiété et symptômes psychosomatiques, etc. (Poikolainen et al., 1994; Poikolainen et al., 2004). Les récents accidents nucléaires à Fukushima au Japon, suite au tsunami, ont montré leur impact sur la santé mentale des résidents, générant une anxiété généralisée et une augmentation du suicide (Le Monde, 2011). Ils ont aussi affecté la perception de la sécurité de ce type d'installations et augmenté les préoccupations entourant I'occurrence d'un désastre nucléaire aux États-Unis (Gallup, 2011), mais aussi dans le monde, Fukushima devenant «le symbole de la menace nucléaire, à partir de laquelle s'est exprimée, à l'échelle mondiale, toute la palette des angoisses, des craintes et des paniques» (Chauvaud, 2011, p. 12).

Les préoccupations écologiques constituent une autre source d'anxiété alimentée par les discours scientifiques et les projections démographiques. Aux États-Unis, les sondages indiquent que les inquiétudes sont élevées, se situant en 2011 à plus de $50 \%$ pour tous les indicateurs choisis, du réchauffement global $(51 \%)$ à la pollution de l'eau potable (77\%). Ces chiffres, bien que très élevés, le sont cependant moins qu'en 2001. Ces appréhensions sont aussi partagées par les Européens (Eurobaromètre spécial, 2008) qui, en 2007, considéraient le changement climatique comme le plus inquiétant ( $57 \%$ ) suivi de la pollution de l'eau (42\%), de l'air (40\%), les catastrophes causées par l'homme (39\%) et par la nature (32\%).

D'autres catastrophes annoncées comme celle de la destruction du monde le 21 décembre 2012 d'après les prédictions mayas, se sont diffusées dans les différents médias et ont suscité des réactions notables. Ces vaticinations ont 
fait l'objet d'un sondage international qui met en évidence un ensemble de croyances et d'anxiétés (Ipsos, 2012). Les réponses indiquent que $14 \%$ des participants sont d'avis qu'ils verront la fin du monde dans leur vie, $10 \%$ sont convaincus de la véracité des prédictions mayas alors que $8 \%$ disent avoir vécu ou vivre des anxiétés et des peurs rattachées à la fin du monde en 2012, mais des différences nationales sont rapportées pour chacune de ces dimensions. Les anxiétés et les peurs sont les plus fortes en Russie, suivie de la Pologne, la Chine, la Turquie et le Japon. Les moins enclins à ce type de réactions proviennent de I'Allemagne, de I'Australie, du Canada et de GrandeBretagne, avec $5 \%$ et moins des répondants, un pourcentage qui représente quand même pour le Canada une population de près de 1,75 million de personnes dont le bien-être pourrait être affecté par ces annonces. Les attentes apocalyptiques contemporaines, sans se situer toujours dans un contexte religieux, mettent cependant en relief certaines lignes de force communes qui démontrent leurs répercussions sur l'imaginaire collectif entourant le destin de notre planète et qui affectent l'état de la santé mentale. Il reste à voir si ces répercussions sont circonstancielles et disparaissent une fois la sensationalité des nouvelles dépassée ou, au contraire, si elles se maintiennent à plus long terme sans nécessairement entraîner le recours aux services de santé.

\section{LES DÉLIRES APOCALYPTIQUES ET DE FIN DE MONDE PARMI LES PATIENTS PSYCHIATRIQUES}

Les délires apocalyptiques et de fin de monde ont aussi été analysés dans le cadre du diagnostic des troubles mentaux et ont été problématisés dès les débuts de la psychiatrie moderne (De Martis, 1965; Rudaleviciene et al., 2008a), en particulier chez les patients souffrant de formes de schizophrénie aux causes encore mal élucidées. Ceux-ci ne semblent plus en contact avec leur environnement et présentent des dysfonctionnements cognitifs (hallucinations visuelles, mais surtout auditives), comportementaux et sociaux souvent associés à des sentiments de persécution exprimés dans des délires. Renvoyant à des perturbations dans le fonctionnement de la pensée, qui peuvent être intermittents ou chroniques, ces délires comprennent des fausses croyances et des raisonnements paralogiques. Depuis l'analyse du cas célèbre du président Schreiber (Freud, 2011) qui mettait en évidence le thème de la fin du monde dans ses délires comme «projection de la catastrophe intérieure» (De Martis, 1965, p. 111), les travaux sur cette problématique montrent l'influence des thèmes apocalyptiques évangéliques qui servent de référence paradigmatique dans le contenu de symptômes psychotiques chez les patients vivant dans les milieux chrétiens (Jaspers, 1962).

Les thèmes de la destruction du monde sans contenu apocalyptique explicite ont aussi été recensés dans la littérature psychiatrique. À la fin des années 1930, Spring (1939) a fait état de références aux tremblements de terre, aux inondations, aux guerres, aux révolutions et aux épidémies qui détruiront la 
planète ou qui affectent des régions et des villes plus localisées. Ces délires, dont les narrativités varient, peuvent donner lieu à des obsessions anxiogènes. Les études plus récentes (Rudalevicience et al., 2008a), menées sur un échantillon de patients souffrant de schizophrénie et résidant à Vilnius en Lituanie, se sont penchées sur la classification du contenu des délires de fin de monde qu'ils rapportent pour dégager trois catégories. La première renvoie aux délires à contenu explicitement religieux faisant appel aux images judéo-chrétiennes issues de la Bible. La fin du monde est interprétée en référence au retour de Jésus-Christ, au Jugement Dernier, etc. La seconde catégorie, définie comme moderne, renvoie à l'interprétation de signes dans I'environnement actuel qui démontreraient la détérioration des systèmes de valeurs (absence d'altruisme et d'amour, criminalité, usage des drogues, terrorisme, rumeurs de guerre, etc.). La troisième classe porte sur des thématiques plus globales (désastres atomiques et autres, collision entre des planètes, explosion de la planète, etc.). La distribution de ces catégories indique que $27,5 \%$ des patients rapportaient des délires de type religieux, $18 \%$ de type moderne, $22,4 \%$ de type global, alors que $32,2 \%$ ne mentionnaient aucun délire de fin de monde. Si les facteurs de scolarité, d'éducation, de lieu de naissance ou d'âge à l'apparition de la maladie n'interviennent pas sur leur distribution, elle varie significativement selon le genre: $32,2 \%$ des femmes rapportant des délires de type religieux (contre 19,1\% de type moderne et $15,1 \%$ de type global) alors que $30,1 \%$ des hommes rapportaient surtout des délires globaux (22,4\% de type religieux et $16,8 \%$ de type moderne). L'importance de la foi religieuse intervient aussi significativement: $75,8 \%$ des répondants pour qui la foi était importante rapportant la croyance en l'imminence de la fin du monde. Parmi les patients pour qui la foi est importante, 32,3\% rapportaient des délires apocalyptiques de type religieux, $19,8 \%$ de type moderne et $21,8 \%$ de type global, alors que $26,2 \%$ ne rapportaient pas ce type de délires. Quant à ceux pour qui la foi n'était pas importante, seulement $8,5 \%$ rapportaient des délires de type religieux et $25,5 \%$ de type global.

Des études comparatives internationales contemporaines ont aussi mis en évidence que la fréquence des délires de fin de monde ne variait pas toujours selon les populations de patients schizophrènes de différents pays, contrairement aux hypothèses plus anciennes de Wetzel (1922), qui suggérait qu'elles seraient plus fréquentes dans les milieux chrétiens. Parmi des patients d'origine japonaise et allemande (Tateyama, 1989), les différences étaient absentes, le pourcentage se situant autour de $5 \%$. Cette convergence entre les deux groupes à cet égard se vérifie de nouveau dans une enquête plus récente (Tateyama et al., 1993). Ces délires peuvent s'accompagner d'idées grandioses et d'identification à des figures religieuses (réincarnation d'archanges, Christ/Messie et à Dieu) (Vardy et Kaplan, 2008; Silva et al., 1997), avec des différences de genre: les hommes se déclarant plus significativement être une divinité que les femmes, qui se prenaient plutôt pour des saintes (Rudaleviciene et al., 2008b). Selon des études de cas psychiatriques, des patients souffrant de délires apocalyptiques prétendent ainsi être 
I'incarnation de l'Antéchrist, comme le rapporte le récit d'un homme de 32 ans, de confession protestante. Convaincu d'être l'antéchrist et Lucifer, faisant explicitement référence à la bataille finale d'Armageddon, il se considérait à la veille de provoquer la destruction d'une partie de l'humanité par ses actions (Silva et al., 1997). Des conduites agressives (viols sous la menace d'une arme) et d'autres formes de violence dirigée contre des personnes (tentative de meurtre, abus sexuel) ont aussi été rapportées chez des patients souffrant de ce type de délires. Cet ensemble de données suggère que les références apocalyptiques, dans leur diversité, occupent une place importante dans les délires de patients souffrant de schizophrénie, mais leur catégorisation ne peut suffire à en comprendre la fonction, l'origine et l'évolution.

\section{CONCLUSIONS}

Cet aperçu met en évidence la diversité des représentations apocalyptiques et de fin de monde qui se retrouvent dans les contenus doctrinaux exprimés dans les délires et les discours. Ceux-ci se fondent sur des croyances religieuses judéo-chrétiennes et en particulier des images fortes et des symboles présents dans le texte de l'Apocalypse de Jean de Patmos, mais ces idéologies se nourrissent aussi des thèmes modernes et post-modernes liés aux idéologies du New Age et aux références à l'imaginaire des contacts extraterrestres. La variabilité de ces discours et leurs métissages suggèrent que les idéologies apocalyptiques se basent sur des formes de bricolage de la pensée qui, malgré des fondements irrationnels, sont susceptibles de séduire et de nourrir la vie affective et relationnelle des participants, le plus souvent impliqués dans des sectes où dominent des formes de contrôle social des adeptes sous l'emprise de leaders charismatiques.

Le profil psychologique de ces leaders suggère la présence d'états mentaux problématiques, incluant le narcissisme et la mégalomanie. Leur identification à des figures divines, spirituelles et religieuses centrales vient confirmer leur statut d'exception, assure leur ascendance sur leurs fidèles et peut les amener à des conduites suicidaires et violentes pouvant aller jusqu'à des actions terroristes sous-tendues par des idéologies qui reflètent des tendances paranoïaques et agressives. La dynamique des sectes met en évidence des rapports complexes entre les leaders et leurs adeptes. Les leaders font appel à des dispositifs d'emprise et de séduction dont il reste à mieux comprendre les modalités à partir de travaux de terrain plus précis pour pouvoir cerner plus justement les répercussions sur la santé mentale. Les conclusions des études à cet égard présentent en effet des perspectives contradictoires qui suggèrent que le type de sectes, leurs idéologies et leurs croyances peuvent intervenir sur le recrutement des participants, tout comme les formes d'organisation sociale, les normes et l'ethos dominant. Ces variations indiquent que l'articulation entre les plans collectif et individuel obéit à des configurations encore mal comprises. 
Les idées apocalyptiques ou de fin de monde se retrouvent aussi à de nombreuses reprises dans I'histoire et la culture occidentales, depuis les millénarismes jusqu'aux préoccupations dérivant des innovations technologiques, des épidémies et de la détérioration catastrophique de l'environnement. Ces éléments jouent aussi sur le niveau d'anxiété des populations, selon des modalités variées en fonction de l'âge et des pays - un état de malaise que I'on retrouve associé aux prédictions de fin de monde pour l'année 2012. Ces peurs sont-elles passagères ou ancrées? Resteront-elles privées ou demanderont-elles une intervention des instances de santé mentale? En dernier lieu, ces types de délires, dans leur diversité de contenu, traditionnel ou plus moderne, semblent avoir une fonction importante dans l'expression des troubles psychotiques liés en particulier à la schizophrénie. Cependant les études dans ce domaine restent encore trop marquées par une perspective classificatoire qui ne peut rendre compte suffisamment de leur fonction dans l'économie psychique des patients. Ces perspectives suggèrent que les idéologies apocalyptiques et les imaginaires de la fin de monde alimentent les délires et les anxiétés qui doivent faire l'objet d'une réflexion attentive de la part des instances en charge de la santé publique afin de mettre en place des stratégies d'éducation, de prévention et de traitement dans ce domaine. Il sera ainsi possible d'éviter des dérives dangereuses et de contribuer à l'amélioration de la santé mentale.

\section{BIBLIOGRAPHIE}

ADITYANJEE (1994). «Jauhar: mass suicide by self-immolation in Waco, Texas», Journal of Nervous \& Mental Disease, vol. 182, p. 727-728.

ARONOFF, J., S.J. LYNN et P. MALINOSKI (2000). «Are cultic environments psychologically harmful?», Clinical Psychological Review, vol. 20, $\mathrm{n}^{\circ} 1$, p. 91-111.

BLACK, A. (1990). «Jonestown - two faces of suicide: a Durkheimian analysis», Suicide and Life Threatening Behavior, vol. 20, n 4, p. 285-306.

BOYER, P. (1984). «From Activism to Apathy: The American People and Nuclear Weapons», The Journal of American History, vol. 70, n 4, p. 821-844.

BUTZ, M. (2000). «The Aum Shinrikyo», http://www.watchman.org/profile/ aumpro.htm. Consulté en août 2012.

CHAUVAUD, F. (2011). L'ennemie intime, Presses universitaires de Rennes.

CNNUS (1999). «Poll Many foresee YK2 cash crunch, few fear doomsday», http://articles.cnn.com/1999-01-10/us/9901_10_y2k.poll_1_extra-cashdoomsday-bank-accounts?_s=PM:US. Consulté en août 2012.

DANZIG, R., M. SAGEMAN, T. LEIGHTON, L. HOUGH, H. YUKI, R. KOTANI et Z.M. HOSFORD (2011). «Aum Shinrikyo insights into how terrorists develop biological and chemical weapons», http://www.cnas.org/files/documents/ publications/CNAS_AumShinrikyo_Danzig_0.pdf. Consulté en août 2012.

DAVIS, W. (2000). «Heaven's Gate: A study of religious obedience», Nova Religio, The Journal of Alternative and Emergent Religions, vol. 3, p. 241267.

DAWSON, L.L. (1999). «When prophecy fails and faith persists: A theoretical overview », Nova Religio, the Journal of Alternative and Emergent Religions, vol. $3, \mathrm{n}^{\circ} 1$, p. $60-82$. 
DEIN, S. et R. LITTLEWOOD (2000). «Apocalyptic suicide», Mental Health, Religion and Culture, vol. 3, $\mathrm{n}^{\circ} 2$, p. 109-114.

DELUMEAU, J. (1978). La peur en Occident, Paris, Hachette.

DE MARTIS D. (1965). «Réflexions sur les délires de négation et de fin de monde», Évolution Psychiatrique, vol. 30, n 1, p. 111-121.

ENCYCLOPÉDIE DE LA MORT (2012). «Suicide collectif», http://agora.qc.ca/ thematiques/mort/dossiers/suicide_collectif. Consulté en août 2012.

EUROBAROMÈTRE SPÉCIAL (2008). «Attitudes des citoyens européens visà-vis de l'environnement», http://ec.europa.eu/public_opinion/archives/ ebs/ebs_295_fr.pdf. Consulté en août 2012.

FREUD, S. (2011). Le président Schreiber. Un cas de paranoïa, Paris, petite Bibliothèque Payot.

GALLUP (2011). «Disaster in Japan Raises Nuclear Concerns in U.S., 16 mars $2011 \gg$, http://www.gallup.com/poll/146660/Disaster-Japan-RaisesNuclear-Concerns.aspx. Consulté en août 2012.

GOUGUENHEIM, S. (1999). Les Fausses terreurs de I'an Mil; attente de la fin des temps ou approfondissement de la foi?, Paris, Éditions Picard.

GRUNSCLOSS, A. (1998). «When we enter into my Father's spacecraft: Cargoistic hopes and millenarian cosmologies in new religious UFO movements», Marburg Journal of religion, vol. 3, $\mathrm{n}^{\circ} 2$, http://archiv.ub. uni-marburg.de/mjr/ufogruen.html. Consulté en août 2012.

HARVEY, J. (2009). «Biography: Jim Jones, leader of the Jonestown massacre», http://www.helium.com/items/1020807-biography-jim-jonesleader-of-the-jonestown-massacre. Consulté en septembre 2012.

HUDSON, R.A. (1999). "The Sociology and Psychology of Terrorism: Who Becomes a Terrorist and Why?», http://www.futurebrief.com/Soc_Psych_ of_Terrorism.pdf. Consulté en août 2012.

INFOSECTE (2003). «Le phénomène des sectes», ministère des Relations avec les citoyens et de l'Immigration. http://infosect.freeshell.org/infocult/ phenomene/phenomene.pdf. Consulté en août 2012.

IPSOS (1999). «Les Français et la peur de I'an $2000 »$, http://www.ipsos.fr/ ipsos-public-affairs/sondages/francais-et-peur-I-2000. Consulté en août 2012.

IPSOS (2012). "One in Seven (14\%) Global Citizens Believe End of the World is Coming in Their Lifetime», http://www.ipsos-na.com/news-polls/ pressrelease. aspx ?id=5610. Consulté en août 2012.

JASPERS, K. (1962). Socrates, Buddha, Confucius, Jesus the paradigmatic individuals, San Diego, New York, London, A Harvest Book.

LANDES, R. (1996). «Apocalyptic Expectations around the Year 1000», http://www.mille.org/scholarship/1000/1000-br.htm. Consulté en août 2012.

LEWIS, J.R. (dir.) (2006). The Order of the Solar Temple: The Temple of Death, Burlington, Ashgate Publishing Company.

LEWIS, J.R. (2003). Legitimating New Religions, Rutgers University Press.

L'EXPRESS (2011). «Seuls $27 \%$ des Franciliens ont peur du sida»,

http://www.lexpress.fr/actualite/sciences/sante/seuls-27-des-franciliensont-peur-du-sida_1055636.html. Consulté en août 2012.

LYONS, L. (2003). «The Gallup Brain: Facing Fear in America, 4 mars», http://www.gallup.com/poll/7903/gallup-brain-facing-fear-america.aspx. Consulté en août 2012. 
LE MONDE (2011). «Le Japon a connu un pic de suicides après la catastrophe du 11 mars $2011 »$,

http://democratie-reelle-nimes.over-blog.com/article-le-japon-a-connu-unpic-de-suicides-apres-la-catastrophe-du-11-mars-2011-101262497.html. Consulté en août 2012.

MUESSE, M.W. (1999). «Religious studies and «Heaven's Gate»: Making the strange familiar and the familiar strange», dans R.T. MCCUTCHEON (dir.), The Insider/Outsider Problem in the Study of Religion, Londres, Cassell, p. 390-394.

NEWPORT, K.G.C. (2006). The Branch Davidians of Waco: The History and Beliefs of an Apocalyptic Sect, Oxford University Press.

OFFICE OF SPECIAL COUNSEL (s.d). «History and Beliefs of the Branch Davidians», http://www.apologeticsindex.org/pdf/history.pdf. Consulté en août 2012.

POKOLAINEN, K., R. KANERVA et J. LÖNGVIST (1994). «Threat of nuclear war increases anxiety and psychosomatic symptoms among adolescents», European Child Adolescent Psychiatry, vol. 3, p. 46-51.

POKOLAINEN, K., T.T. AALTO-SETÄLÄ, A. TUULIO-HENRIKSSON, M. MARTTUNEN et J. LÖNGVIST (2004). «Fear of nuclear war increases the risk of common mental disorders among young adults: a five-year followup study», BMC Public Health, 30 septembre, $4: 42$.

PRÉVOST, J.-P. (1999). «Vers une résurgence des millénarismes? Les mouvements apocalyptiques contemporains», Religiologiques, vol. 20, p. $153-164$.

ROBBINS, T. (1986). «Religious mass suicide before Jonestown: The Russian old believers», Sociological Analysis, vol. 47, n 1, p. 1-20.

ROSENBERG, J. (s.d.). «Jim Jones and the Peoples Temple», http:// history1900s.about.com/od/people/p/jimjones.htm. Consulté en septembre 2012.

RUDALEVICIENE, P., T. STOMPE, A. NARBEKOVAS et R . BUNEVICIUS (2008a). «Influence of culture on the world end (apocalyptic) delusions», World Cultural Psychiatry Research Review, vol. 3, n² 2, p. 96-105.

RUDALEVICIENE, P., T. STOMPE, A. NARBEKOVAS, N. RASKAUSKIENE et R. BUNEVICIUS (2008b). «Are religious delusions related to religiosity in schizophrenia?», Medicina, vol. 44, n 7, p. 529-535.

SCHUYLER, P.D. et S. TRINH (2000). Apocalypse Observed: Religious Movements and Violence in North America, Europe, and Japan, London, Routledge.

SENATE GOVERNMENT AFFAIRS PERMANENT SUBCOMMITTEE ON INVESTIGATIONS (1995). «Global Proliferation of Weapons of Mass Destruction: A Case Study on the Aum Shinrikyo», http://www.fas.org/ irp/congress/1995_rpt/aum/index.html. Consulté en août 2012.

SIEBER, O. (2010). «Grippe A: I'apocalypse n'aura pas lieu», La Revue Nouvelle, février, $\mathrm{n}^{\circ} 2$, http://www.revuenouvelle.be/rvn_abstract. php3 ?id_article=1789. Consulté en août 2012.

SILVA, J.A., G.B. LEONG et R. WEINSTOCK (1997). «Violent behaviors associated with the antichrist delusion», Journal of Forensic Sciences, vol. 42, n०6, p. 1058-1061.

SPRING, W.J. (1939). «Observations on world destruction fantasies», Psychoanalytic Quarterly, vol. 8, p. 48-56. 
TATEYAMA, M. (1989). «"Delusion of world destruction" (Wetzel). Comparative study between Japan and West Germany», Psychopathology, vol. 22, $\mathrm{n}^{\circ} 6$, p. 289-294.

TATEYAMA, M., M. ASAI, M. KAMISADA et al. (1993). "Comparison of schizophrenic delusions between Japan and Germany», Psychopathology, vol. 26, n० 3-4, p. 151-158.

TORKGHASHGHAEI, E. (2009). L'univers apocalyptique des sectes. Une approche pluridisciplinaire, Paris, L'Harmattan.

VARDY, M.M. et B.M. KAPLAN (2008). «Christ/Messiah delusions revisited: Toward an anthropological definition of religious delusions », Psychoanalytic Review, vol. 95, n³, p.473-487.

WETZEL, A. (1922). «Das weltuntergangserlebnis in der schizophrenie», $Z f$. d. g. Neurologie Und Psychiatrie, vol. 28, p. 403-428.

WHITSETT, D. et S.E. KENT (2003). «Cults and families», Families in Society, vol. 84, n० 4, p. 491-502.

WRIGHT, S.A. (dir.) (1996). Armageddon in Waco: Critical Perspectives on the Branch Davidian Conflict, Chicago, University of Chicago Press.

ZELLER, B.E. (2010). «Extraterrestrial biblical hermeneutics and the making of Heaven's Gate», Nova Religio: The Journal of Alternative and Emergent Religions, vol. 14, n², p. 34-60. 